\title{
Oral Endotracheal Tube Cuff Pressure in Patients Undergoing Mechanical Ventilation Admitted to the Intensive Care Unit
}

\author{
Zohreh Maleki', Mohammadreza Dinmohammadi ${ }^{*}$, Taraneh Naghibi ${ }^{2}$ \\ ${ }^{1}$ Department of Critical Care Nursing, School of Nursing and Midwifery, Zanjan University of Medical Sciences, Zanjan, Iran \\ ${ }^{2}$ Department of Anesthesiology, Education and Treatment Center of Mousavi, Zanjan University of Medical Sciences, Zanjan, Iran
}

\section{A BSTRACT}

Introduction: Regular monitoring and maintaining oral endotracheal tube (ETT) cuff pressure in the normal range in patients undergoing mechanical ventilation in order to maintain adequate ventilation and reduce the risk of complications is essential. This study was aimed to determine the oral ETT cuff pressures in mechanically ventilated patients were admitted to the intensive care unit. Materials and Methods: This cross-sectional study conducted between February 2015 and June 2015. Fifty patients with oral ETT were admitted to the intensive care unit were selected. Pressure of ETT cuff was measured by a standard calibrated manometer. Data were analyzed by SPSS ver 22. Results: The Mean of oral ETT cuff pressure was $53.40 \pm 25.42 \mathrm{~cm}$ of $\mathrm{H}_{2} \mathrm{O}$. In $76 \%$ of cases, the cuff pressure was higher than $30 \mathrm{~cm}$ of $\mathrm{H}_{2} \mathrm{O}$. There was no correlation between factors related to patients and ETT cuff pressure. Only significant correlation between the ETT cuff pressure and body temperature was observed. Conclusion: Cuff pressure is often higher than safe limits and regular monitoring of the ETT cuff pressure and preventing its consequences are much recommended.

\section{Key words:}

1. Intubation

2. Critical Care

3. Critical Illness

*Corresponding Author: Mohammadreza Dinmohammadi

E-mail:mdinmohammadi@zums.ac.ir 


\title{
فشار كاف لولهُ داخل تراشةُ دهانى در بيماران تحت تهويةٔ مكانيكى بسترى در بخش مراقبتهاى ويزه
}

\author{
زهره ملكى'، محمدرضا دين محمدى"'، ترانه نقيبى؟ \\ 'حروه يرستارى مراقبتهاى ويزّه، دانشكده يِرستارى و مامايى، دانشعاه علوم يزشكى زنجان، زنجان، ايران \\ ‘َّروه بيهوشى، مركز آموزشى و درمانى آيت الله موسوى، دانشعاه علوم يزشكى زنجان، زنجان، ايران
}

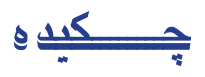

مقدمه: نظارت منظمه و حفظ فشار كاف لوله داخل تراشأ دهانى در محدودئ نرمال در بر بيماران تحت

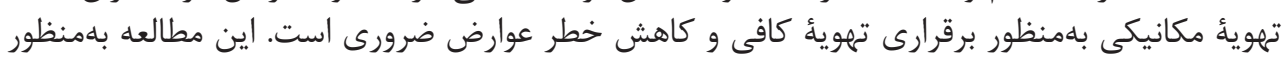

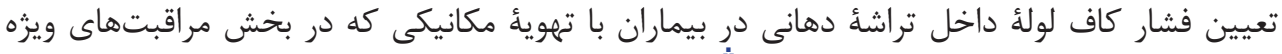

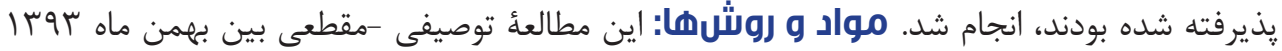

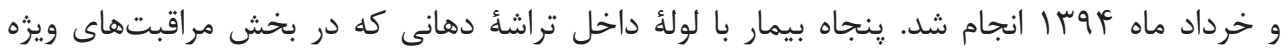

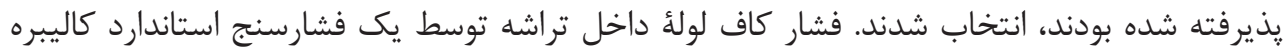

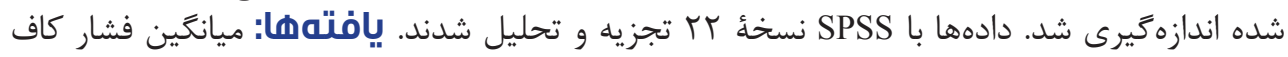

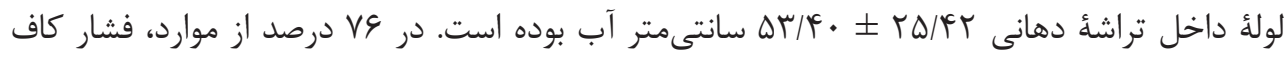

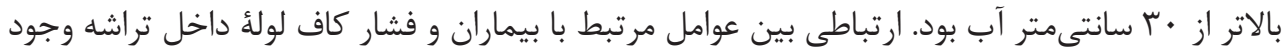

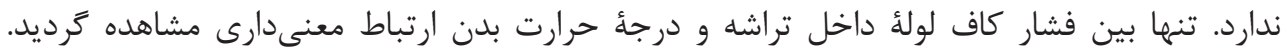

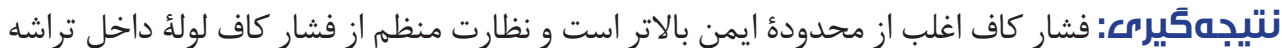
و جلوگيرى از عواقب آن بسيار توصيه مى أفود.

ا. ا. لوله كذارى داخل تراشه

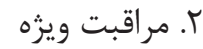

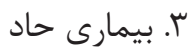

" نويسنده مسئول: محمدرضا دينمحمدى : آدم آدرس الكترونيكى: mdinmohammadi@zums.ac.ir 


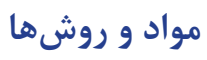

اين مطالعهُ توصيفى -مقطعى بر روى • له نفر از بيماران داراى لولئ

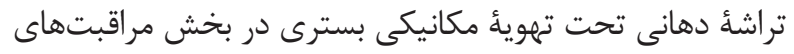

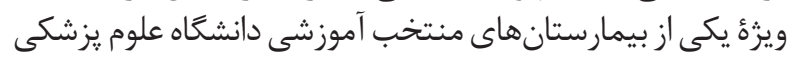

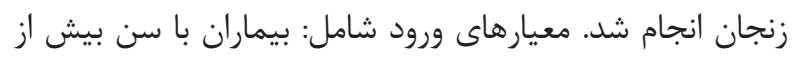

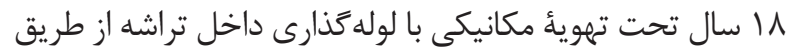

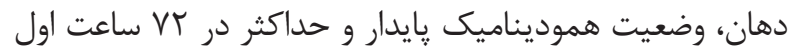

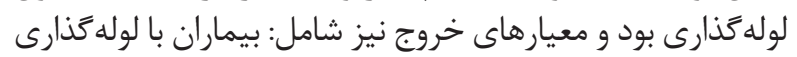

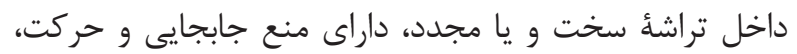

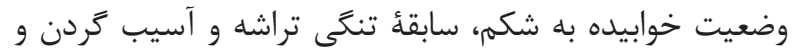

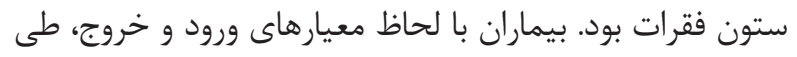

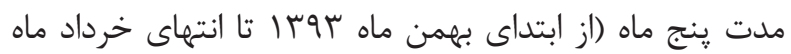

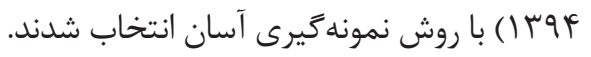

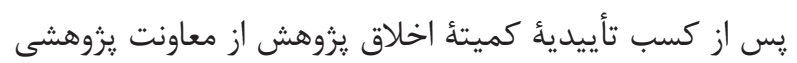

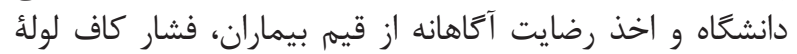

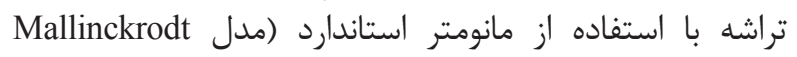
النازه Medical, Athlone, Ireland

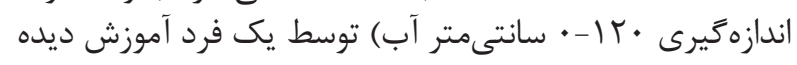

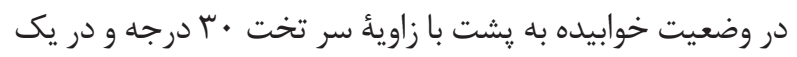

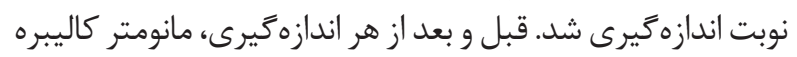

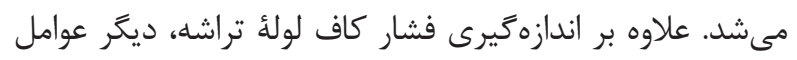

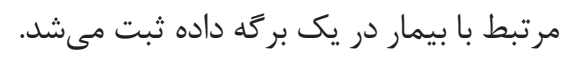

تجزيه و تحليل دادهها

دادههاى مطالعه با استفاده از شاخصهاى آنآ آمارى (فراوانى،

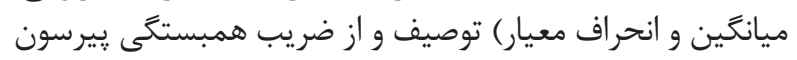

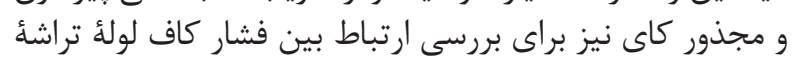

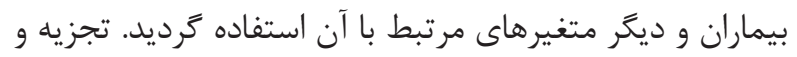

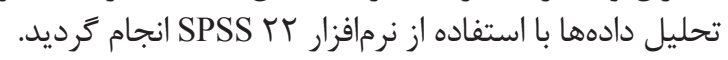

يافتهها

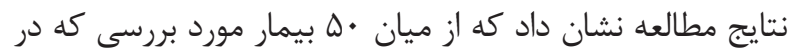

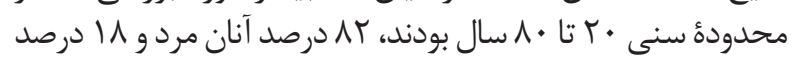

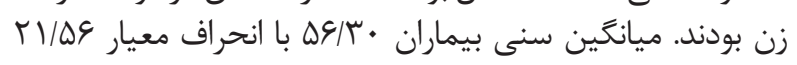

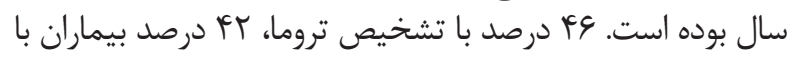

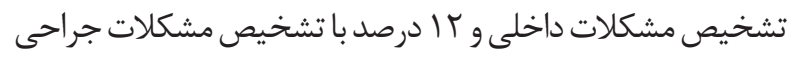

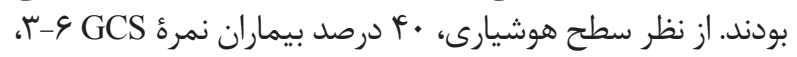

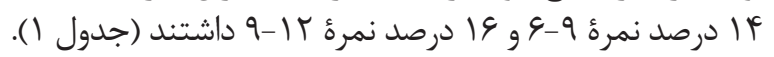

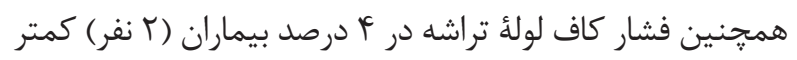

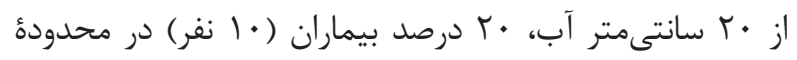

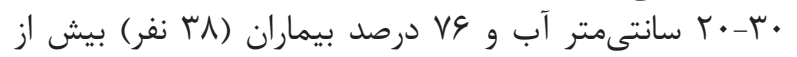

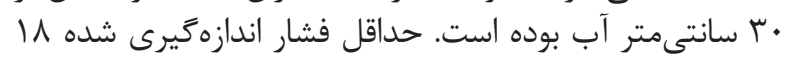

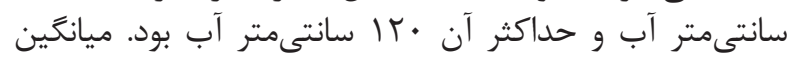

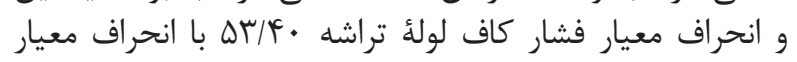

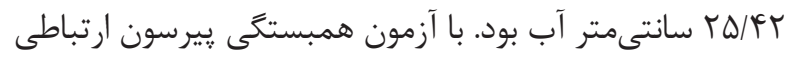

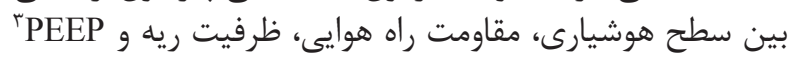

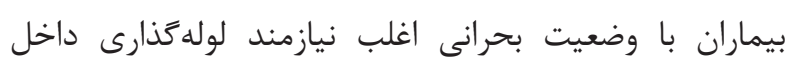

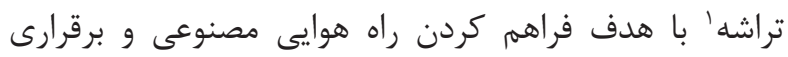

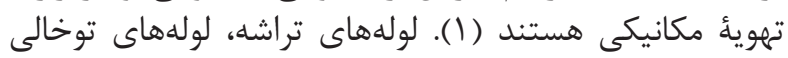

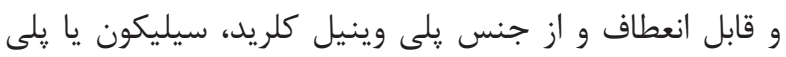

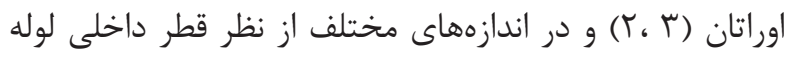

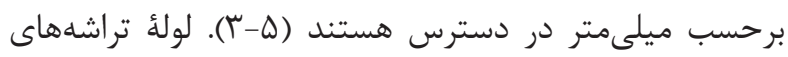

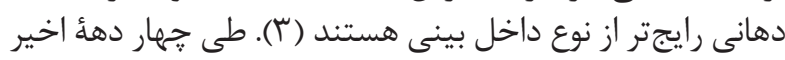

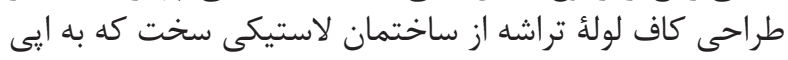

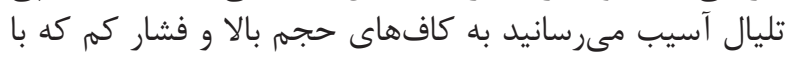

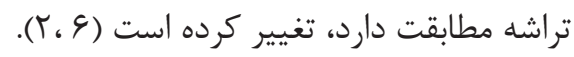

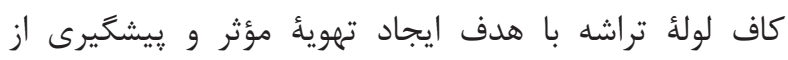

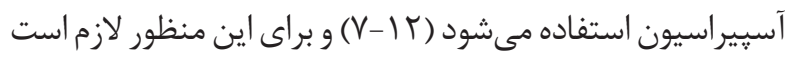

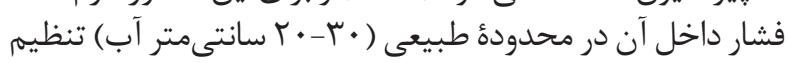

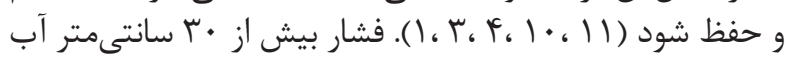

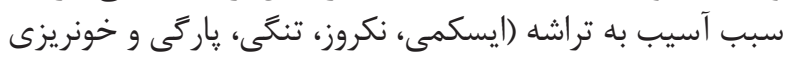

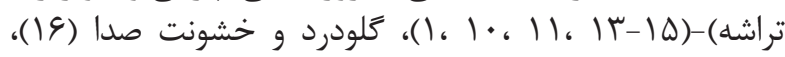

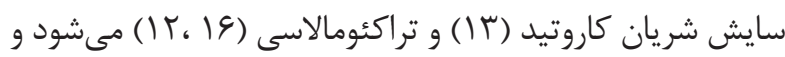
ميزان آسيب با كذشت زمارونيد (مان افزايش مراكومالاسى (V)

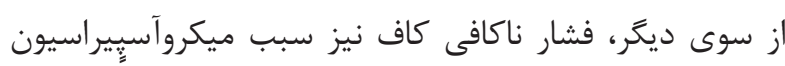

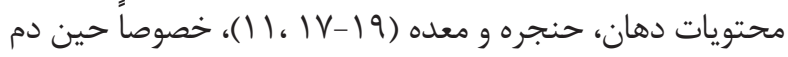

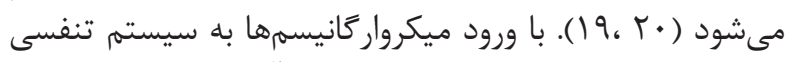

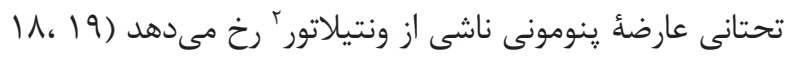

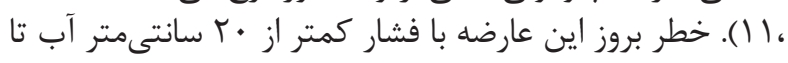

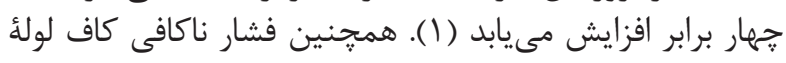

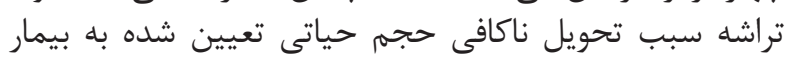

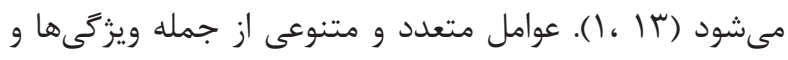

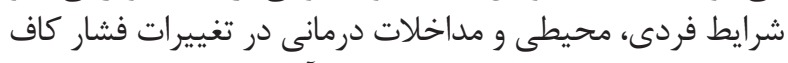

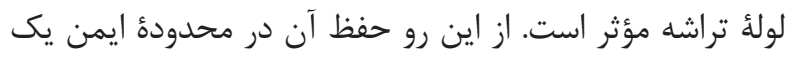

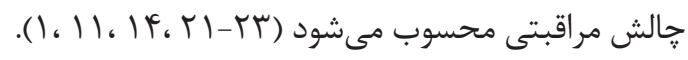

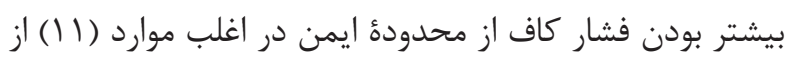

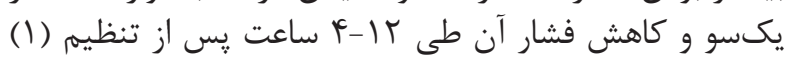

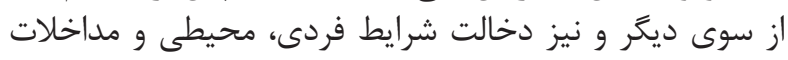

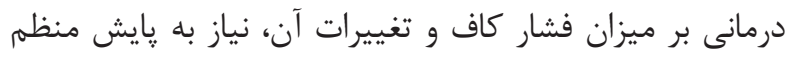

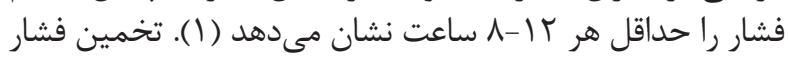

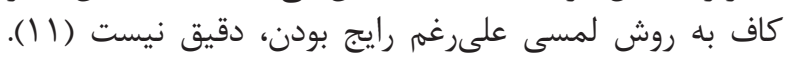

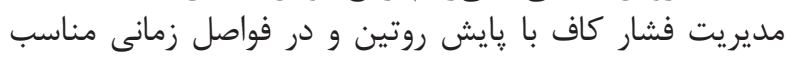

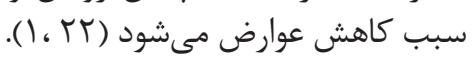

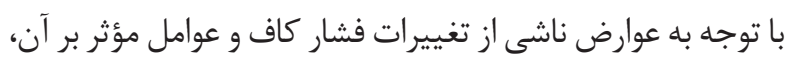

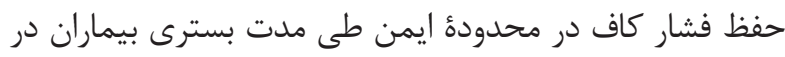

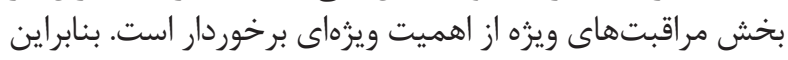

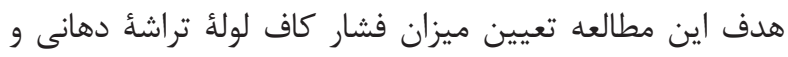

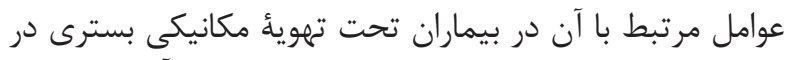

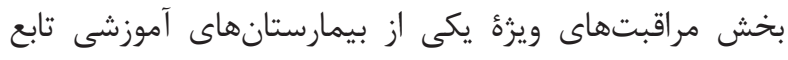
دانشخاه علوم يزشكى زنجان بودي.

\footnotetext{
${ }^{1}$ Intubation

${ }^{2}$ Ventilator

${ }^{3}$ Positive end-expiratory pressure
} 


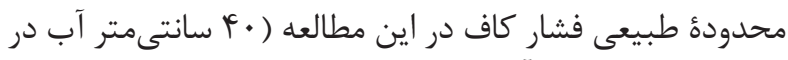

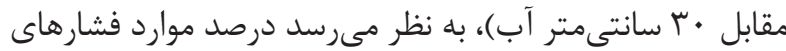

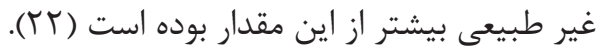
همجنين در مطالعهُ عمادى و همكاران كه بر روى بيماران تحت

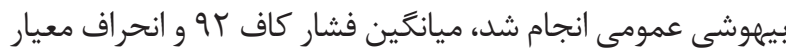

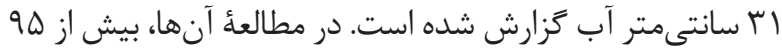

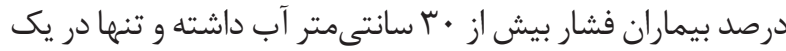

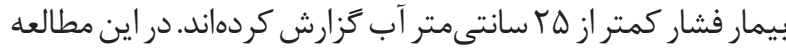

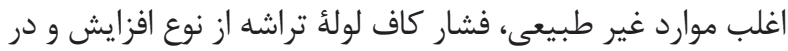

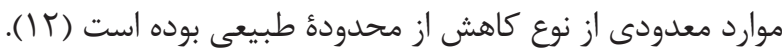

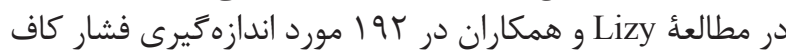

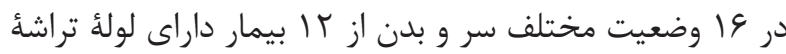

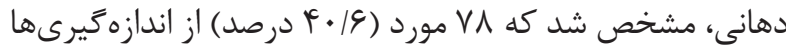

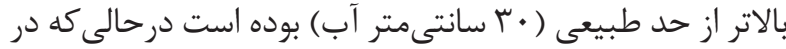

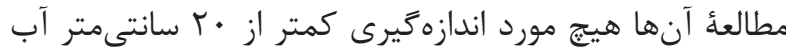

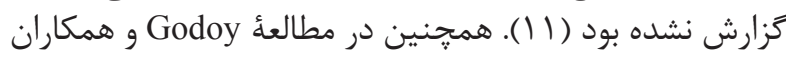

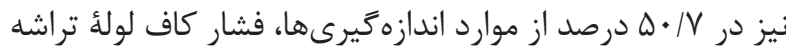

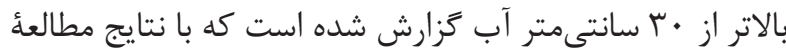

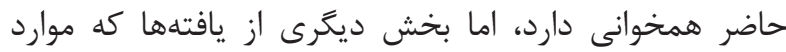

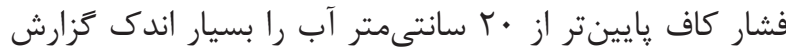

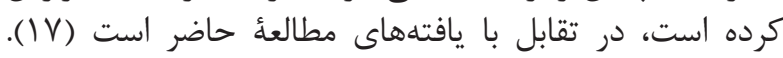

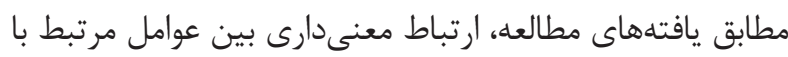

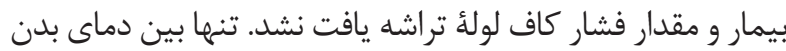

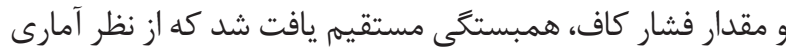

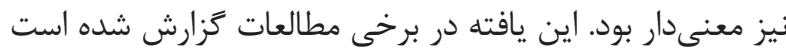
(IF، IV)

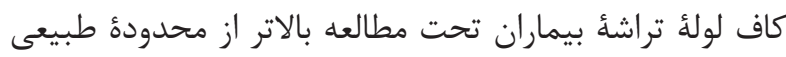

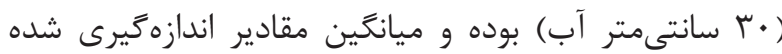

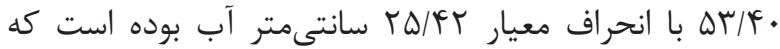

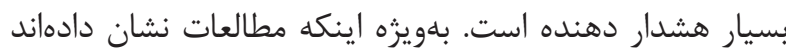

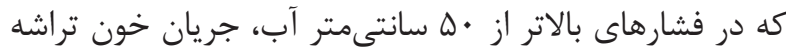

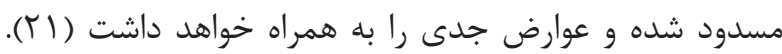
متخصصين و يرستاران بخش مراقبت إنهاى ويره بايد از مديريت

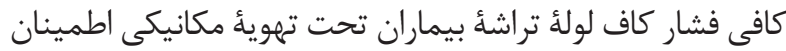

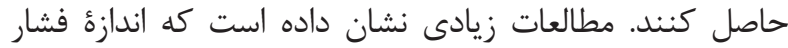

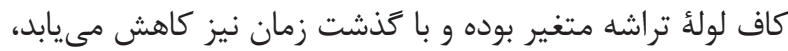

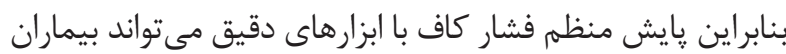

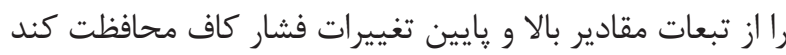

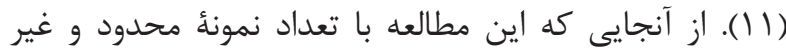

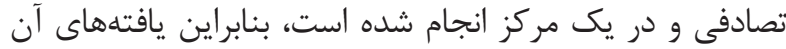

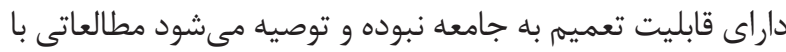

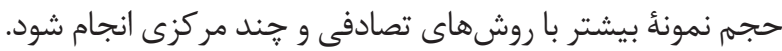

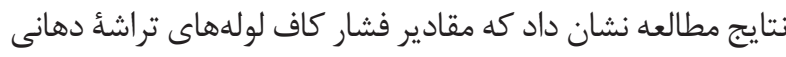

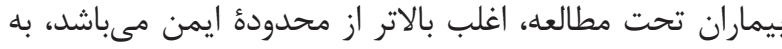

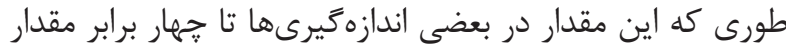

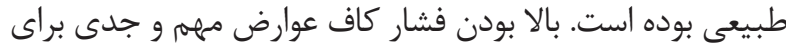
بيمار به همراه دارد، از اين رو يايش منظهم فيشار كاف كارض ماف لولئ تراشه براي

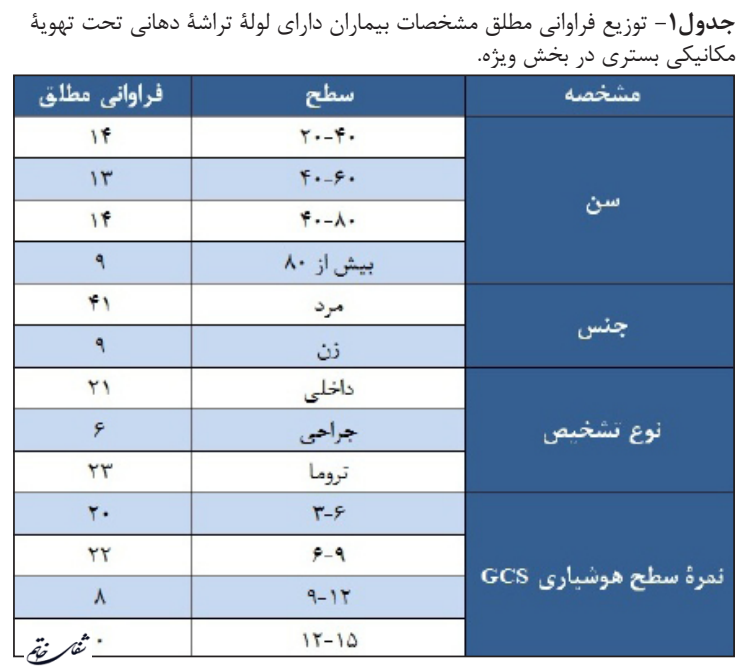

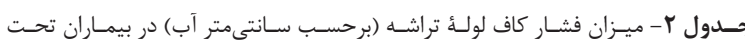

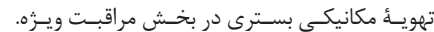

\begin{tabular}{|c|c|c|c|}
\hline ميانكين 土|نحراف معيار & فراوانى نسبى & فراوانى مطلق & فشار كاف فراوانى \\
\hline \multirow{3}{*}{$\Delta r / F \cdot \pm r \Delta / F r$} & r & r & كمتر از • r r \\
\hline & $r$. & 1. & $r \cdot-r$. \\
\hline & ve & ru & بيشتر از ·r ب ب \\
\hline
\end{tabular}

با فشار كاف لولأ تراشه وجود نداشت. همبستخى مستقيم اندكى دئى

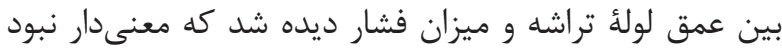

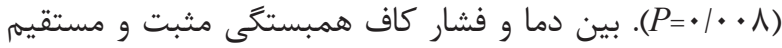

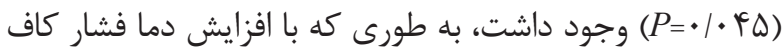

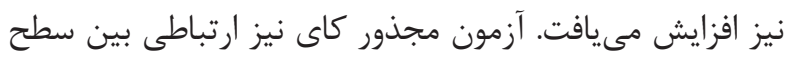

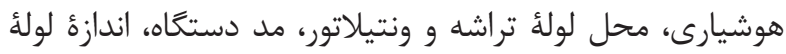

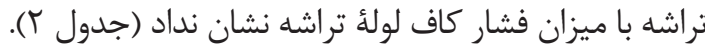

\section{بحث و نتيجه بيرى}

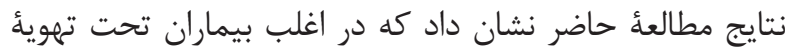

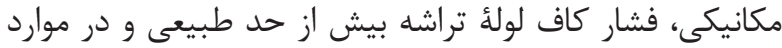

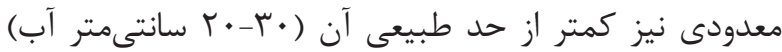

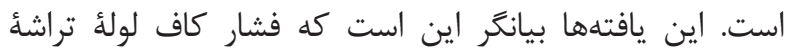

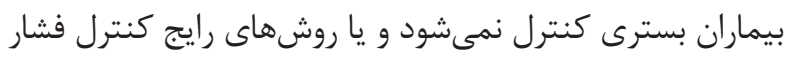

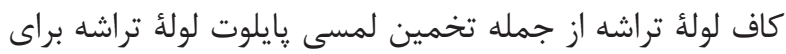

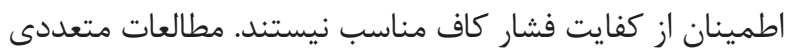

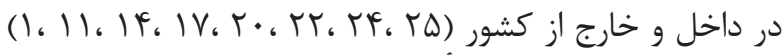

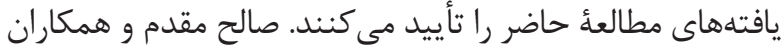

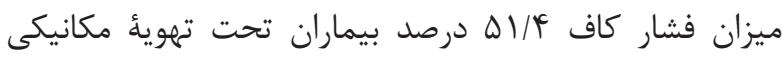

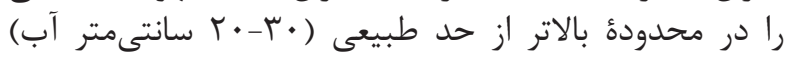

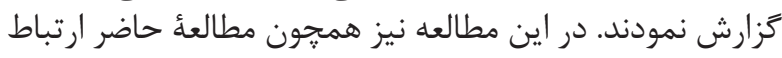

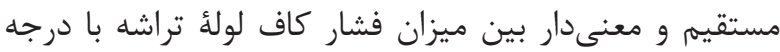

حرارت بيمار وجود داشت (4 (l) (1).

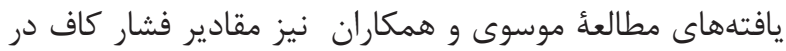

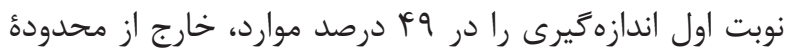

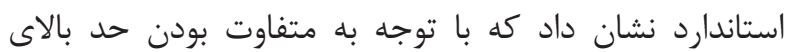


علوم يزشكى زنجان با كد اخلاق (ZUMS.REC.1393.172)

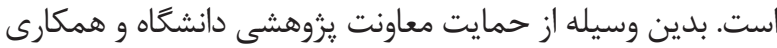

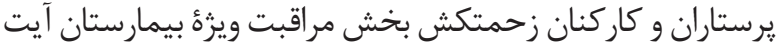

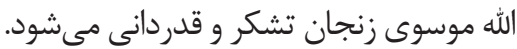

1. Sole ML, Penoyer DA, Su X, Jimenez E, Kalita SJ, Poalillo E, et al. Assessment of endotracheal cuff pressure by continuous monitoring: a pilot study. Am J Crit Care. 2009; 18(2): 133-43.

2. Louise R, Redl L. Minimal occlusive volume cuff inflation: a survey of current practice. Intensive Crit Care Nurs. 2008; 24(6): 359-65.

3. Chulay M, Burnes S. AACN essentials of critical care nursing. $2^{\text {nd }}$ ed. New York. McGraw-Hill Education. 2010.

4. Urden LD, Stacy KM, Lough ME. Critical care nursing, diagnosis and management. $7^{\text {th }}$ ed. Critical Care Nursing: Elsevier/Mosby. 2013.

5. Shiri H, Nicravanmofrad M. Principals of intensive care in CCU, ICU, and Dialysis. Noor-e-Danesh. 2004.

6. Braz JR, Camacho Navarro LH, Takta LH, Nascimento HJunior P. Endotracheal tube cuff presure: need for precise measurement. Sao Paulo Med J. 1999; 117(6): 243-7.

7. Bernon JK, McGuire C, Carrara H, Lubbe D. Endotracheal tube cuff pressures-the worrying reality: a comparative audit of intra-operative versus emergency intubations. S Afr Med J. 2013; 103(9): 641-3.

8. Bolzan WD, Guizilini S, Carvalho A, Paola A, Gomes W. Endotracheal tube cuff pressure assessment maneuver induces drop of expired tidal volume in the postoperative of cronary artery bypass grafting. J Cardiothorac Surg. 2012; 7(23): 1-5.

9. Maboudi A, Abtahi H, Hosseini M, Tamadon A, Safavi E. Accuracy of endotracheal tube cuff pressure adjustment by fingertip palpation training of intensive care units nursing. Iran Red Crescent Med J. 2012; 15(5): $381-4$.

10. Ansari 1, Bohluli B, Mahaseni H, Valaei N, SadrEshkevari P, Rashad A. The effect of endotracheal tube cuff pressure control on postextubation throat pain in orthogenathic surgeries: a randomized double-blind controlled clinical trial. Br J Oral Maxillofac Surg. 2014; 52(2): 140-3.

11. Lizy C, Swinnen W, Labeau S, Poelaert J, Vogelaers D, Vandewoude K, et al. Cuff pressure of endotracheal

$$
\begin{aligned}
& \text { و يیشگيرى از تبعات ناشى از آن به طور جدى توصيه مىشود. }
\end{aligned}
$$

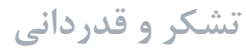

$$
\begin{aligned}
& \text { اين مطالعه بخشى ازطرح تحقيقاتى مصوب معاونت يثوهشى دانشگاه } \\
& \text { منابع }
\end{aligned}
$$

tubes after changes in body position in critically ill patients treated with mechanical ventilation. Am J Crit Care. 2014; 23(1): e1-e8.

12. Emadi SA, Zamani A, Nasiri E, Khademlo M, Mohammad T. Assessment of endotracheal tube cuff pressure after tracheal intubation during general anaesthesia. Journal of Mazandaran University of Medical Sciences. 2010; 20(76): 9-13.

13. Nikbakhsh N, Alijanpour E, Mortazavi Y, Organji N. Evaluation of tracheal tube pressure complication in ICU patients of Shahid Beheshti hospital. Journal of Babol University of Medical Sciences. 2010; 12(2): 30-4.

14. Salehmoghaddam AR, Malekzadeh J, Mesbahi Z, Esmaeily H. Relationship between inspiratory oxygen fraction and temperature in mechanically ventilated patients with endotracheal tube cuff pressure. Journal of Police Medicine. 2012; 1(2): 81-7.

15. Nseir S, Jaillette E. Continuous control of tracheal cuff pressure and ventilator-associated pneumonia. Reanimation. 2013; 22(3): 245-9.

16. Art M, Rettig T, Vries J, Wolfs J, Veld B. Mantaining endotracheal tube cuff pressure at $20 \mathrm{mmHg}$ to prevent dysphagia after anterior cervical spin surgery; protocol of a double-blind randomised controlled trial. BMC Musculoskelet Disord. 2013; 14(280): 1-4.

17. Godoy A, Vieira R, Captani E. Endotracheal tube cuff pressure altration after changes in position in patients under mechanical ventilation. J Bras Pneumol. 2008; 34(5): 294-7.

18. Sengupta P, Sessler D, Maglinger P, Wells S, Vogt A, Durrani J, et al. Endotracheal tube cuff pressure in three hospitals, and the volume requred to produce an appropriate cuff pressure. BMC Anesthesiol. 2004; 4(8): 1-6.

19. Rose L, Redl L. Survey of cuff management practices in intensive care units in Australiane and New Zealand. Am J Crit Care. 2008; 17(5): 427-35.

20. Duguet A, D'Amico L, Biondi G, Prodanovic H, Gonzalez-Bermejo J, Similowski T. Control of tracheal cuff pressure:a pilot study using a pneumatic device. Intensive Care Med. 2007; 33(1): 128-32. 
21. Sole ML, Su X, Talbert S, Penoyer DA, Kalita $\mathrm{S}$, Jimenez $\mathrm{E}$, et al. Evaluation of an intervention to maintain endotracheal tube cuff pressure within therapeutic range. Am J Crit Care. 2011; 20(2): 10917.

22. Mousavi SAJ, Niakan lahiji M, Akhovatian F, Moradi Moghadam O, Valizade Hassanlouei MA. An investigation of endotracheal tube cuff pressure. Bimonthly Official Publication Medical Daneshvar. 2009; 17(83): 43-8.

23. Minonishi T, Kinoshita H, Hirayama M, Kawahito S, Azma T, Hatakeyama N, et al. The supine-to-prone position change induces modificationof endotracheal tube cuff pressure accompanied by tube displacement. J Clin Anesth. 2013; 25(1): 28-31.

24. Chapman J, Pallin D, Ferrara L, Mortell S, Pliakas $\mathrm{J}$, Shear M, et al. Endotracheal tube cuff pressures in patients intubated before transport. Am J Emerg Med. 2009; 27(8): 980-2.

25. Soleimani M, Rajabi MR, Fakhr-movahedi A, Ghods AA. Effects of endotracheal tube cuff pressure regulation with minimal occlusion volume on incidence of ventilatior-associated pneumonia. Journal of Semnan Medical Sciences Univesity. 2014; 15(2): 68-75. 